\title{
Long-Read Sequencing Emerging in Medical Genetics
}

\author{
Tuomo Mantere ${ }^{1,2}$, Simone Kersten ${ }^{1,3,4}$ and Alexander Hoischen ${ }^{1,3,4 *}$ \\ ${ }^{1}$ Department of Human Genetics, Radboud University Medical Center, Nijmegen, Netherlands, ${ }^{2}$ Laboratory of Cancer \\ Genetics and Tumor Biology, Cancer and Translational Medicine Research Unit and Biocenter Oulu, University of Oulu, Oulu, \\ Finland, ${ }^{3}$ Department of Internal Medicine, Center for Infectious Diseases (RCl), Radboud University Medical Center, \\ Nijmegen, Netherlands, ${ }^{4}$ Radboud Institute for Molecular Life Sciences, Radboud University Medical Center, Nijmegen, \\ Netherlands
}

\section{OPEN ACCESS}

Edited by:

H. Steven Wiley,

Pacific Northwest National Laboratory

(DOE), United States

Reviewed by:

Rui Chen,

Baylor College of Medicine,

United States

Adam Ameur,

Uppsala University, Sweden

*Correspondence:

Alexander Hoischen alexander.hoischen@radboudumc.nl

Specialty section: This article was submitted to Genomic Assay Technology, a section of the journal

Frontiers in Genetics

Received: 25 October 2018 Accepted: 18 April 2019

Published: 07 May 2019

Citation:

Mantere T, Kersten S and Hoischen A (2019) Long-Read

Sequencing Emerging in Medical Genetics. Front. Genet. 10:426. doi: 10.3389/fgene.2019.00426
The wide implementation of next-generation sequencing (NGS) technologies has revolutionized the field of medical genetics. However, the short read lengths of currently used sequencing approaches pose a limitation for the identification of structural variants, sequencing repetitive regions, phasing of alleles and distinguishing highly homologous genomic regions. These limitations may significantly contribute to the diagnostic gap in patients with genetic disorders who have undergone standard NGS, like whole exome or even genome sequencing. Now, the emerging long-read sequencing (LRS) technologies may offer improvements in the characterization of genetic variation and regions that are difficult to assess with the prevailing NGS approaches. LRS has so far mainly been used to investigate genetic disorders with previously known or strongly suspected disease loci. While these targeted approaches already show the potential of LRS, it remains to be seen whether LRS technologies can soon enable true whole genome sequencing routinely. Ultimately, this could allow the de novo assembly of individual whole genomes used as a generic test for genetic disorders. In this article, we summarize the current LRS-based research on human genetic disorders and discuss the potential of these technologies to facilitate the next major advancements in medical genetics.

Keywords: long-read sequencing, next-generation sequencing, medical genetics, structural variation, tandem repeat expansion, phasing, pseudogenes

\section{INTRODUCTION}

Since its introduction over a decade ago, next-generation sequencing (NGS) of DNA has become a routine diagnostic tool for modern medical genetics and revolutionized the discovery of novel Mendelian disease genes (Gilissen et al., 2011). Due to the high-throughput nature and low costs of NGS compared to Sanger sequencing, previous single gene approaches have largely been replaced by gene panels and whole exome sequencing (WES), allowing better clinical diagnostics (Gilissen et al., 2011; Goodwin et al., 2016). However, despite the numerous advancements that NGS has conferred, many studies are still hindered by the short-read lengths ( $\sim 150-300 \mathrm{bp})$ that the current NGS technologies are bound to use in order to preserve high read quality (Goodwin et al., 2016). Issues arising from the use of short reads can mainly be pinned down to the highly repetitive and complex nature of the human genome (Feuk et al., 2006; de Koning et al., 2011; Treangen and Salzberg, 2011). It has been shown that despite the use of sophisticated bioinformatic algorithms 
it is often impossible to accurately map, or even assemble, short reads originating from regions harboring structural variation (SV), repetitive sequences, extreme guanine-cytosine (GC) content, or sequences with multiple homologous elements within the genome (Salzberg and Yorke, 2005; Treangen and Salzberg, 2011). This introduces errors in calling genetic variants and inability to capture certain genomic regions (Ashley, 2016). Furthermore, with short-read NGS (SR-NGS) the variant phasing information is often lost (Delaneau et al., 2013) and the data analysis is highly dependent on the reference genomes, which are known to be imperfect (Genovese et al., 2013). The dependency on the reference genome is especially problematic for the detection of SVs at complex genomic regions that can be highly individual- or population-specific (Chaisson et al., 2015b).

Genetics research has always been strongly driven by novel sequencing technologies, first by Sanger sequencing and later followed by NGS (Shendure et al., 2017). With the recently demonstrated success in identifying previously intractable DNA sequences and closing gaps in the human genome assemblies (Chaisson et al., 2015a; Seo et al., 2016; Shi et al., 2016; Jain et al., 2018), long-read sequencing (LRS) technologies hold the promise to overcome specific limitations of NGS-based investigations of human diseases. The main advantage of LRS compared to other platforms stems from the use of long-reads ( $>10$ kilobase [kb] on average), originating from single DNA molecules (van Dijk et al., 2018). Moreover, the sequencing process occurs in real-time and both the sequencing and library preparation are conducted without the need of PCR amplification, therefore being free from any PCR related bias (Schadt et al., 2010). The absence of PCR leaves the DNA in its native state, enabling LRS technologies to directly detect base modifications, such as methylation (Flusberg et al., 2010; Laszlo et al., 2013).

Attributable to these features, LRS has the potential to grow into a technology that is used not only to produce high-quality genome assemblies (i.e., the platinum human reference genome) (Chaisson et al., 2015a; Pollard et al., 2018), but also to capture clinically relevant genomic elements which are problematic for conventional approaches (summarized in Figure 1). This has been shown in several studies by (1) identifying disease causing SVs that SR-NGS might not detect, (2) directly sequencing repeat expansions and regions with extreme GC-content, (3) resolving variant phasing and (4) distinguishing a gene of interest from its pseudogenes (Table 1). Importantly, recent studies have indicated that LRS technology may play an important role in discovering novel pathogenic mutations in human diseases with previously unknown genetic causes (Aneichyk et al., 2018; Ishiura et al., 2018; Zeng et al., 2018) and open up unprecedented opportunities to investigate transcriptomics (Byrne et al., 2017). In the future, this technology could ultimately allow whole genome sequencing (WGS) and even de novo assembly capturing all variant types present in individual genomes, independently of the reference genome limitations (Chaisson et al., 2015b). In this review, we summarize the LRS-based research of human genetic diseases and discuss the future promise of these technologies to enable the next major advancements in medical genetics. We focus on the prevailing true LRS methods that have been commercially released so far; single molecule real-time (SMRT) sequencing by
Pacific Biosciences (PacBio) and nanopore sequencing by Oxford Nanopore Technologies Inc. (ONT) (Clarke et al., 2009; Eid et al., 2009) (Box 1 for technical summary).

\section{OVERCOMING CURRENT LIMITATIONS IN MEDICAL GENETICS}

Whole exome sequencing (WES), mainly using Illumina platforms, is often used as a first-tier test for many genetic diseases. This has been very successful and has brought genetic testing and diagnostics into a whole new era (Gilissen et al., 2011). However, for many patients that have undergone diagnostic WES, or even WGS, the underlying cause of their disease remains unsolved (Gilissen et al., 2014). Curiously, the recent WGS studies applying LRS have revealed that each human genome harbors $>20,000$ of SVs ( $>50 \mathrm{bp}$ ) and additional thousands of indels ( $<50 \mathrm{bp}$ ), spanning dozens of megabases (Mbs), that have largely remained undetected with conventional SR-NGS (Chaisson et al., 2015b, 2019; Pendleton et al., 2015; Seo et al., 2016; Shi et al., 2016; Huddleston et al., 2017; De Coster et al., 2018). In patients with suspected genetic diseases, such hidden SVs and indels may well disrupt relevant genes or cause a dosage change for dosage-sensitive genes. Furthermore, NGS methods heavily rely on PCR, which leads to GC-content dependent coverage bias (Benjamini and Speed, 2012). Low or zero coverage at genomic locations with extreme GC-contents are estimated to span $>150 \mathrm{Mb}$ of gene-rich regions (Genomes Project Consortium et al., 2012) and some of those may as well account for a proportion of the hidden genetic variation underlying human diseases. Among the approaches that are based on SR-NGS, the PCR-free library preparation methods provide the most uniform genome coverage and may allow sequencing of some of the previously difficult regions (Dolzhenko et al., 2017). However, SR-NGS always requires a PCR-step in the template amplification, e.g., bridge-PCR, which may still introduce a sequencing bias.

In contrast to the contemporary SR-NGS methods, LRS technologies require no PCR amplification and sequencing is performed on single molecules instead of colonies or clusters of amplified DNA molecules, resulting in a more evenly distributed coverage (Schadt et al., 2010). This, together with longer reads that can span over challenging genomic regions and SVs, may offer an unprecedented view on previously poorly characterized genomic locations (Chaisson et al., 2015a; Jain et al., 2018) and eventually improve the variant calling of population-scale sequencing data (Ameur et al., 2018a). While increases in the throughput and lower prices may in the future allow more longread WGS for individual patients, targeted approaches are already demonstrating the value of LRS for medical genetics (Tables 1, 2) (Ameur et al., 2018b).

\section{Detection and Characterization of Structural Variation}

High-depth sequencing by NGS is a reliable and cost-efficient method for detecting single nucleotide variants (SNVs) and small indels (Ashley, 2016; Goodwin et al., 2016). However, 


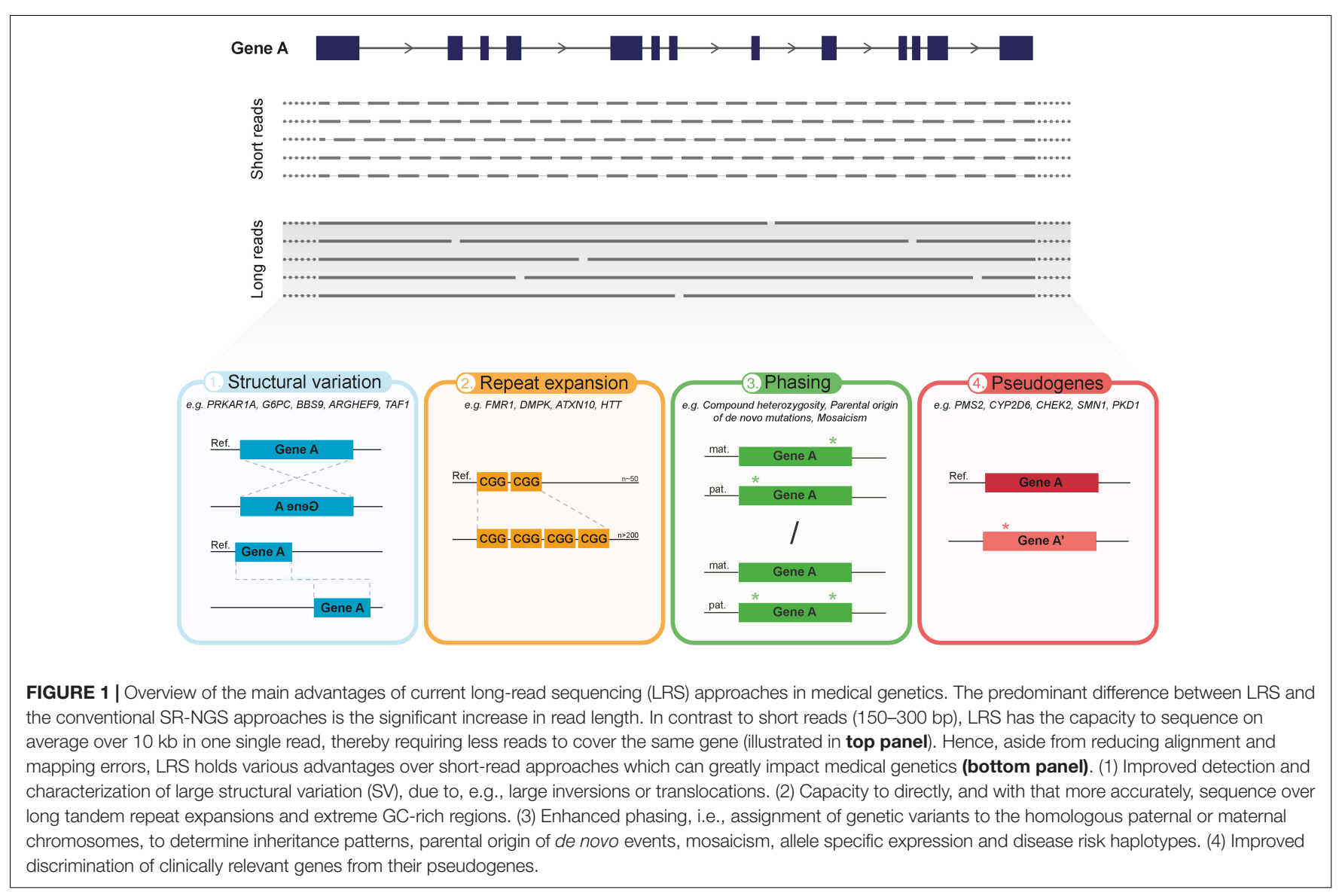

in the detection of larger genomic deletions, duplications and rearrangements, SR-NGS approaches often lack sensitivity, show excess of false positives and misinterpret complex SVs (Tattini et al., 2015; Ashley, 2016; Huddleston et al., 2017; Sedlazeck et al., 2018b). Yet, SVs $>50$ bp in size are an important source of genetic variation, accounting for the greatest number of divergent bases across human genomes (Alkan et al., 2011; Sudmant et al., 2015; Chaisson et al., 2019). Moreover, SMRT sequencing of a haploid human genome has shown that as much as $89 \%$ of the identified variation, consisting mainly of SVs, had been missed in the 1000 genomes project (Huddleston et al., 2017). Similarly, a sevenfold increase in SV detection was achieved by a multiplatform approach, including LRS, compared to standard SRNGS methods (Chaisson et al., 2019). It is likely that this gain in SV detection sensitivity is due to the different sequencing technology itself, as this benefits from longer fragments, no PCR, and less sequence bias.

The detection of SVs by SR-NGS is indirect and based on read depth coverages and use of paired-end reads, for which the approximate length between read pairs is known (Tattini et al., 2015). For copy number variants (CNVs), the excess of reads can indicate amplification whereas the loss of reads is suggestive for a deletion. Rearrangements can be discovered using pairedend reads by investigating deviations in the expected distances and orientations between read pairs (Tattini et al., 2015). In contrast to SR-NGS, the long reads obtained with PacBio or ONT sequencers are more likely to span SV breakpoints, or often the entire SV event, with high-confidence alignments (Huddleston et al., 2017; De Coster et al., 2018; Sedlazeck et al., 2018b). Furthermore, longer reads can be more confidently aligned to repetitive sequences that often mediate the formation of SVs (Sedlazeck et al., 2018b). Long reads also allow better distinction of haplotypes, further contributing to the accuracy of analyzing SVs (Seo et al., 2016; Cretu Stancu et al., 2017). Other methods, such as microarray copy number profiling and karyotyping, have traditionally been used to detect disease causing SVs. However, with these approaches it is not possible to map small or copybalanced SVs, and they do not provide accuracy at base-pair resolution, nor the possibility to resolve complex SVs (Alkan et al., 2011). Thus, LRS can substantially increase the resolution and reliability of SV detection and mapping.

Recently, Cretu Stancu et al. (2017) proposed LRS as an alternative approach for genome-wide detection of clinically relevant SVs and used ONT-WGS to analyze two patients with congenital abnormalities caused by de novo chromothripsis rearrangements. The authors demonstrated that long reads, even with a relatively low coverage of $16 \times$, are superior to short reads (WGS with average coverage of $\sim 30 \times$ ) in detecting and mapping the breakpoints of the rearrangements. The improved phasing capability with long reads also enabled the determination of the parental origin of these de novo events (Cretu Stancu et al., 2017). Other studies have demonstrated the potential benefits of LRS 
TABLE 1 | Human genetic diseases investigated with LRS technologies.

\begin{tabular}{|c|c|c|c|}
\hline Phenotype & Technology & Finding & Reference(s) \\
\hline \multicolumn{4}{|c|}{ Identification and fine-mapping of structural variation } \\
\hline Developmental disorder & ONT & $\begin{array}{l}\text { Complex rearrangements } \\
\text { (chromothripsis) }\end{array}$ & Cretu Stancu et al., 2017 \\
\hline Carney complex & SMRT & Large deletion (PRKAR1A) & Merker et al., 2018 \\
\hline Bardet-Biedl syndrome & SMRT & Large deletion (BBS9) & Reiner et al., 2018 \\
\hline Glycogen storage disease IA & ONT & Large deletion (G6PC) & Miao et al., 2018 \\
\hline Developmental disorder & ONT & Chromosomal translocation & Dutta et al., 2018 \\
\hline X-linked Parkinsonism & SMRT and $10 \times$ genomics & SVA insertion (TAF1) & Aneichyk et al., 2018 \\
\hline \multicolumn{4}{|c|}{ Sequencing over tandem repeat expansion loci } \\
\hline Fragile- $X$ & SMRT & $\begin{array}{l}\text { Repeat expansion length and } \\
\text { interruption motifs (FMR1) }\end{array}$ & $\begin{array}{l}\text { Loomis et al., 2013; Ardui et al., 2017; } \\
\text { Ardui et al., 2018b }\end{array}$ \\
\hline SCA10 and Parkinson's disease & SMRT & $\begin{array}{l}\text { Repeat expansion length and } \\
\text { interruption motifs (ATXN10) }\end{array}$ & $\begin{array}{l}\text { Schule et al., 2017; McFarland et al., } \\
2015\end{array}$ \\
\hline ALS and FTD & SMRT and ONT & Repeat expansion length (C9orf72) & Ebbert et al., 2018 \\
\hline Huntington's disease & SMRT & $\begin{array}{l}\text { Repeat expansion length and somatic } \\
\text { variability }(H T T)\end{array}$ & Hoijer et al., 2018 \\
\hline Myotonic dystrophy 1 & SMRT & $\begin{array}{l}\text { Repeat expansion length, interruption } \\
\text { motifs and somatic variability (DMPK) }\end{array}$ & Cummings et al., 2017 \\
\hline BAFME and FCMTE & SMRT and ONT & $\begin{array}{l}\text { Novel repeat expansion loci (SAMD12, } \\
T N R C 6 A \text {, and RAPGEF2) }\end{array}$ & Ishiura et al., 2018; Zeng et al., 2018 \\
\hline Alzheimer's disease & ONT & $\begin{array}{l}\text { ABCA7 repeat expansion length and } \\
\text { alternative sequence motifs }\end{array}$ & De Roeck et al., 2017 \\
\hline \multicolumn{4}{|l|}{ Resolving allele phasing } \\
\hline KID syndrome & SMRT & Revertant mosaicism (CX26) & Gudmundsson et al., 2017 \\
\hline Treacher Collins and Noonan syndrome & SMRT & $\begin{array}{l}\text { Parental origin of de novo mutations } \\
\text { (TCOF1 and PTPN11) }\end{array}$ & Wilbe et al., 2017 \\
\hline \multicolumn{4}{|l|}{ Discriminating pseudogenes } \\
\hline ADPKD & SMRT & Pseudogene discrimination (PKD1) & Borras et al., 2017 \\
\hline Primary immunodeficiency & SMRT & Pseudogene discrimination (IKBKG) & Frans et al., 2018 \\
\hline Drug metabolism & SMRT and ONT & $\begin{array}{l}\text { Pseudogene discrimination and allele } \\
\text { phasing (CYP2D6) }\end{array}$ & $\begin{array}{l}\text { Ammar et al., 2015; Qiao et al., 2016; } \\
\text { Buermans et al., } 2017\end{array}$ \\
\hline
\end{tabular}

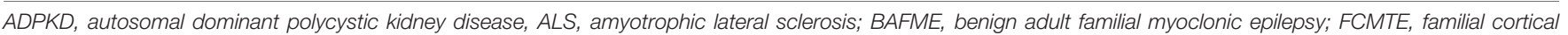

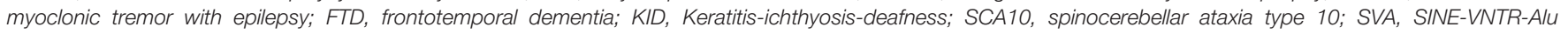
retrotransposon.

over standard SR-NGS in clinical diagnostics to detect pathogenic SVs. In a study by Merker et al. (2018), a patient with clinically diagnosed Carney complex had previously received negative test results from diagnostic gene panel sequencing and WGS. In contrast, low-coverage SMRT-WGS successfully identified the causative heterozygous deletion of over $2 \mathrm{~kb}$ overlapping the first exon of PRKAR1A (Merker et al., 2018). Similarly, Miao et al. (2018) explored ONT-WGS to confirm the diagnosis for a patient with recessive glycogen storage disease by identifying a secondhit ( $\sim 7 \mathrm{~kb}$ deletion) in G6PC, which had remained undetected in previous WES analysis. Together, these studies indicate that LRS has the ability to surpass conventional NGS-methods in the detection of SVs and yield clinical diagnoses at a molecular level in previously unsolved cases.

The fine-mapping of SVs at single-nucleotide resolution is important for patients in which translocation or inversion breakpoints disrupt disease causing genes (Chen et al., 2010). For this, long reads are highly beneficial and may render mapping by laborious amplicon-based Sanger sequencing obsolete, as demonstrated by Reiner et al. (2018) who used SMRT to finemap BBS9 deletion in a patient with Bardet-Biedl syndrome.
In another diagnostic case-study, ONT-WGS was able to track down the exact breakpoints of a reciprocal translocation, which led to the identification of disrupted ARHGEF9 gene in a girl with intellectual disability (Dutta et al., 2018).

In addition to the improved identification and mapping of SVs affecting known disease genes, we are now seeing the first discoveries of novel disease genes harboring SVs that are characterized with LRS technologies. As an outstanding example, an unbiased de novo assembly using LRS (SMRT and synthetic longs reads with $10 \times$ Genomics) was important for the identification of SINE-VNTR-Alu insertion in TAF1, a newly discovered gene for X-linked Dystonia Parkinsonism (Aneichyk et al., 2018). While multiple studies indicate that LRS is superior to SR-NGS in the discovery of SVs, it is also shown that once the alternate allele is resolved, many of these events $(\sim 61 \%)$ can be genotyped with high accuracy and lower costs using SR-NGS (Huddleston et al., 2017). This shows that part of the missed SVs are present in the SR-NGS data and therefore improved software may still provide a more complete picture of genomes sequenced with short reads. It is noted that the current cut-off of $50 \mathrm{bp}$ for SVs is arbitrary; indels $<50 \mathrm{bp}$ are also identified by LRS 


\section{BOX 1 | Technical summary of LRS technologies. \\ True versus synthetic LRS}

The current LRS technologies can be divided into true LRS and synthetic LRS technologies. In synthetic LRS approaches the long stretches of DNA are linked by molecular barcodes, which is followed by sequencing with conventional short-reads and in silico construction of the original DNA molecule (Goodwin et al., 2016). Usually these methods rely on serial-dilutions of very long DNA fragments and physical separation before library preparation in which molecule specific barcodes are added (Kitzman et al., 2011; Peters et al., 2012; Kuleshov et al., 2014). Similar technologies have recently been further optimized and commercialized by 10x Genomics Inc. (Weisenfeld et al., 2017). These technologies have already provided haplotype aware SR-WGS (Zheng et al., 2016) and improved SV calling from SR-WGS data (Nazaryan-Petersen et al., 2018; Marks et al., 2019). While synthetic long-reads leverage advantages of LRS, some short-read issues may persist, e.g., PCR-bias and intra-read complexity may not always be fully resolved.

\section{SMRT sequencing by PacBio}

As a first commercial platform for LRS, PacBio released the RS sequencer in 2011, which was followed by RSII in 2013 and Sequel in 2015. SMRT technology is based on special flow cells harboring individual picolitre-sized wells with transparent bottoms. Each of the wells, referred to as zero mode waveguides (ZMW), contain a single fixed polymerase at the bottom (Levene et al., 2003; Ardui et al., 2018a). This allows a single DNA molecule, which is circularized in the library preparation (i.e., the SMRTbell), to progress through the well as the polymerase incorporates labeled bases onto the template DNA. Incorporation of bases induces fluorescence that can be recorded in real-time through the transparent bottoms of the ZMW (Levene et al., 2003; Ardui et al., 2018a; Pollard et al., 2018). The average read length for SMRT was initially only $\sim 1.5 \mathrm{~Kb}$, and with reported high error rate of $\sim 13 \%$ characterized by false insertions (Carneiro et al., 2012; Quail et al., 2012). However, the errors are randomly distributed across the reads (Eid et al., 2009) and high consensus sequences can be obtained with sufficient read depths (Roberts et al., 2013; Hebert et al., 2018). Also, for a single molecule, with $\sim 10 \mathrm{~kb}$ read length, each nucleotide position in a $1 \mathrm{~kb}$ amplicon can be read $\sim 10$ times using circular consensus sequence (CCS) method, rendering it unlikely that a same random mistake would occur in multiple reads (Travers et al., 2010; Hestand et al., 2016). Since its introduction, the read length and throughput of SMRT technology have substantially increased. Throughput can reach $>10$ Gb per SMRT cell for the Sequel machine, while the average read length for both RSIl and Sequel is $>10 \mathrm{~kb}$ with some reads spanning $>100 \mathrm{~kb}$ (van Dijk et al., 2018). Still, the persisting downsides for this technology are the high cost/throughput ratio and the requirement of relatively high amounts of high quality DNA as starting material (Ardui et al., 2018a; van Dijk et al., 2018).

\section{Nanopore sequencing by ONT}

As an alternative, scientist have since many years aimed to use biological or synthetic nanopores to read the sequence of DNA molecules (Kasianowicz et al., 1996; Butler et al., 2008). In 2015, nanopore sequencing was commercially introduced by ONT with a portable MinION sequencer, which was followed by more high-throughput desktop sequencers GridION and PromethION. The basic principle of nanopore sequencing is to pass a single strand of DNA molecule through a nanopore which is inserted into a membrane, with an attached enzyme, serving as a biosensor (Deamer et al., 2016). Changes in electrical signal across the membrane are measured and amplified in order to determine the bases passing through the pore in real-time. The nanopore-linked enzyme, which can be either a polymerase or helicase, is bound tightly to the polynucleotide controlling its motion through the pore (Deamer et al., 2016; Pollard et al., 2018). For nanopore sequencing, there is no clear-cut limitation for read length, except the size of the analyzed DNA fragments. On average, ONT single molecule reads are $>10 \mathrm{~kb}$ in length but can reach ultra-long for some individual reads lengths of $>1 \mathrm{Mb}$ surpassing SMRT (Jain et al., 2018). Also, the throughput per run of ONT GridlON and PromethION sequencers are higher than for PacBio (up to $100 \mathrm{~Gb}$ and 6 Tb per run, respectively) (van Dijk et al., 2018). The raw reads have high error rates similar to SMRT dominated by false deletions and in particular homopolymer errors (Jain et al., 2015; Menegon et al., 2017). Currently, the errors are more systematic, and are related to the length and type of the DNA fragment in the nanopore itself, and thus may not all be overcome by just increasing the coverage as with SMRT sequencing (Krishnakumar et al., 2018).

\section{The main limitations of current LRS technologies}

Library preparation: for optimal LRS, fresh material or even intact cells are required. The DNA isolation protocols that are needed and the handling of ultra-long high molecular weight DNA require improvements.

Error rate: both LRS technologies still have a higher error rate compared to SR-NGS. SMRT sequencing may overcome this with CCS also for long-insert libraries (Wenger et al., 2019). It remains to be seen whether this is sufficient for all variant types including SNVs and indels. So far, ONT has more systematic error-profile, which may be more challenging to overcome.

Costs: while LRS is still more expensive than SR-NGS, recent advancements in throughput may offer even lower prices. ONT's PromethION already offers 30x coverage WGS for less than 1,000 dollars, and PacBio's $8 \mathrm{M}$ chip should also significantly reduce the price per human genome. It is also important to realize that the pricings are constantly changing in this rapidly developing field:

https://docs.google.com/spreadsheets/d/1GMMfhyLKO-q8Xklo3YxIWaZA5VVMuhU1kg41g4xLkXc/htmlview?hl=en_GB (documentation of updated sequencing costs by Dr. A Vilella).

Data analysis: both raw data analysis as well as mapping and variant calling tools are much less mature for LRS than SR-NGS but are constantly being improved (Sedlazeck et al., 2018a).

and their frequency in WGS is even much higher than for the $>50$ bp SVs (Chaisson et al., 2019). In addition to PacBio and ONT, synthetic LRS (i.e., by $10 \times$ Genomics) can be highly useful in SV detection (Nazaryan-Petersen et al., 2018; Marks et al., 2019). Uncovering SVs and indels comprehensively holds the potential to unravel a number of novel disease causing mutations underlying human diseases.

\section{Sequencing Tandem Repeat Expansions}

A short tandem repeat is a region of genomic DNA with multiple adjacent copies of short (1-6 bp) sequence units. These repeat regions are highly mutable due to replication errors that occur during cell divisions and to date over 30 human diseases known to be caused by tandem repeat expansions (Tang et al., 2017) or repeat contractions (Lemmers et al., 2018). Since their discovery, the research of tandem repeat expansions has been limited by the overall incompliance of these genomic elements to standard molecular techniques like cloning, PCR and sequencing (Loomis et al., 2013). Most of the disease causing expansions are longer than the currently used NGS reads, making it virtually impossible to accurately assemble those (Treangen and Salzberg, 2011; Lee et al., 2016; Sedlazeck et al., 2018a). Multiple studies have now shown that LRS technologies are well suited to transcend through these long, often GC-rich, repeat expansions (Table 1). This not only allows the direct detection of expansion lengths, but 
also the intra-molecule sequence variation, which might provide clinically relevant additional information (Ardui et al., 2018b; Cumming et al., 2018; Hoijer et al., 2018; McFarland et al., 2015).

The first tandem repeat associated disease studied by LRS was Fragile-X (Loomis et al., 2013), which is caused by CGG-repeat expansion in the 5'UTR of the FMR1 gene (Kremer et al., 1991). With standard DNA sequencing technologies, i.e., SR-NGS and Sanger sequencing, it has been impossible to directly sequence the expanded CGG-repeats, consisting of > 200 units at full mutation range (Nolin et al., 2003; Peprah, 2012). SMRT technology enabled for the first time to completely sequence expanded full mutation FMR1 alleles, up to 750 CGG-repeats, which translates to $>2 \mathrm{~kb}$ of $100 \%$ CGG-repeat DNA (Loomis et al., 2013). The obtained sequencing data and phasing information further allowed to define the presence of 'AGG' interruptions, which affect the risk of a premutation to expand into a full mutation in the following generation (Nolin et al., 2015; Ardui et al., 2017). The clinical use of this information has been largely neglected due to technical limitations, which can now be overcome with LRS (Ardui et al., 2018b). Genetic variation within repeat expansion loci that are causative for other diseases, such as myotonic dystrophy 1 (DM1) and spinocerebellar ataxia 10 (SCA10), have also been investigated with LRS technology (Cumming et al., 2018; McFarland et al., 2015). In DM1, SMRT sequencing detected de novo repeat interruptions at DMPK expansion locus, associated with reduced somatic instability of the repeat expansion and therefore mild or even absent clinical features (Cumming et al., 2018). Moreover, McFarland et al. (2015) demonstrated that SMRT sequencing could identify interruption motifs, potentially acting as phenotypic modifiers, within the tandem repeat expansion locus in ATXN10 of SCA10 patients.

The presented studies on repeat expansions rely on PCRbased target enrichment, which may complicate the analysis due to the occurrence of PCR stutter, chimeric molecules, and false insertions or deletions of the repeat units (Laver et al., 2016; Tsai et al., 2017; Hoijer et al., 2018). Therefore, it has been proposed that the optimal approach to study these repetitive regions would be to sequence single DNA molecules without any prior PCR amplification (Hoijer et al., 2018). For this, Tsai et al. (2017) have developed CRISPR/Cas9-based amplificationfree target enrichment method (No-Amp targeted sequencing, Table 2), which was used to capture repeat the expansion locus in Huntingtin (HTT), responsible for Huntington's disease (Hoijer et al., 2018). This allowed the retrieval of detailed sequence information and assessment of somatic variability of repeat elements without the interference of PCR stutter. In addition, No-Amp approach has been utilized to successfully sequence across C9orf72 and ATXN10 repeat expansion loci in patients with frontotemporal dementia or Parkinson's disease, respectively (Schule et al., 2017; Ebbert et al., 2018). These studies indicate that No-Amp Targeted sequencing could provide an optimal targeted solution to study different repeat elements that are recalcitrant to PCR-based methods.

In the era of CRISPR/Cas9 genome editing, LRS has found use in monitoring the efficiency of editing challenging genomic regions. Dastidar et al. (2018) excised a disease causing tandem repeat expansion in the DMPK locus in DM1 patient-derived cell lines and used SMRT sequencing to monitor the efficiency of the excision. In the future, LRS could be more broadly implemented in genome editing of challenging regions. Moreover, with the capability of directly sequencing through long tandem repeats, LRS technology is already accelerating the discovery and characterization of novel repeat expansions linked to human diseases. So far, this has been demonstrated in familial forms of epilepsy, bipolar disorder and schizophrenia (Ishiura et al., 2018; Song et al., 2018; Zeng et al., 2018). It is noted that, while the current approaches still largely rely on targeted enrichment of suspected repeat expansion loci, ultimately the analysis of all repeat-expansions genome-wide will be retrieved directly by WGS with long reads (De Roeck et al., 2018; Chaisson et al., 2019).

\section{Haplotype Resolution With Long Reads}

Phasing refers to the means of assigning genetic variants to the homologous paternal and maternal chromosomes. In medical genetics, phasing is very important for understanding, e.g., inheritance patterns, parental origin of de novo mutations, mosaicism, allele-specific expression and disease risk-haplotypes (Tewhey et al., 2011). However, in order to directly resolve the haplotype of two heterozygous SNVs they would need to be covered by the same molecule (i.e., read). With SR-NGS this is usually only the case for a limited number of variants (20-30\% of substitutions), despite the use of paired-end reads (Goldmann et al., 2016). Therefore, phasing has largely been based on parental genotypes and statistical imputation (Tewhey et al., 2011) or, in some cases, laborious physical separation of entire chromosomes (Fan et al., 2011). Now, with the substantially longer read lengths, LRS technology enables to directly phase variants multiple kbs apart (Laver et al., 2016) or even assemble and phase complex genomic regions, such as the major histocompatibility complex, in full-length in a diploid human genome (Jain et al., 2018).

Knowing whether two variants in the same gene are in cis or trans can be crucial for diagnostic purposes, especially for compound heterozygosity, which is a commonly observed phenomenon underlying recessive Mendelian disorders (Tewhey et al., 2011). By using LRS, two SNVs in the same gene can be directly phased without the need of testing the parents (Laver et al., 2016) or if one of the two mutations has occurred de novo and the parental genotypes cannot resolve the phase in the offspring (Neveling et al., 2012). In pre-implantation genetic diagnostics (PGD), LRS can be applied to determine the parental origin of pathogenic de novo mutations by simply sequencing across the adjacent variants (Wilbe et al., 2017). Resolving the parental origin of de novo mutations is needed for estimating the recurrence risk of a genetic disease in the case of germline mosaicism (Dimitriadou et al., 2017). LRS could also be used in PGD to directly phase parental genotypes for genome-wide haplotype reconstruction of a single cell via discrete SNP genotypes (i.e., haplarithmisis) (Zamani Esteki et al., 2015). Moreover, phasing with long reads offers an accurate method to study mosaicism. This has recently been shown by Gudmundsson et al. (2017) who used SMRT to resolve allele phasing in an exceptional case of keratitis-ichthyosis-deafness 
TABLE 2 | Different applications of LRS technology.

\section{LR-WGS}

SMRT-WGS

ONT-WGS

\section{Targeted LRS}

LR-PCR amplicon sequencing

Hybridization-based capture

No-Amp targeted SMRT sequencing

CATCH for ONT sequencing

ONT Read until selective sequencing

\section{LR-RNA-sequencing}

SMRT-IsoSeq

Direct ONT RNA-seq

R2C2 method for ONT
De novo assembly and reference-based WGS with focus on structural variant calling (Chaisson et al., 2015a; Seo et al., 2016; Shi et al., 2016).

De novo assembly and reference-based WGS utilizing ultra-long reads to improve phasing and close gaps in the reference genome (Jain et al., 2018).

Commonly used targeted approach with standard LR-PCR amplification of the target region followed by SMRT amplicon sequencing (Borras et al., 2017; Frans et al., 2018)

As for SR-NGS, hybridization-based target capture can be applied for LRS (Wang et al., 2015). Protocols are available for different vendors of bait-libraries.

A standard PacBio SMRTbell library is created and a Cas9 guide RNA is designed adjacent to the region of interest. Digestion with Cas9 breaks open the SMRTbell molecules to enable ligation with a capture adapter. SMRTbell molecules that contain the capture adapter are enriched on magnetic beads and prepared for SMRT Sequencing (Tsai et al., 2017; Hoijer et al., 2018).

CATCH (Cas9-assisted targeting of chromosome segments) is based on targeted fragmentation of DNA in vitro by Cas9, followed by separation of the target region from the rest of the genomic DNA by pulsed field gel electrophoresis and DNA isolation from the gel (Jiang et al., 2015; Gabrieli et al., 2018)

Real-time data analysis that enables the selection of specific DNA molecules for sequencing by reversing the driving voltage across individual nanopores: this enables to proceed to sequence only molecules that are recognized to originate from a certain chromosome or region of interest (Loose et al., 2016).

The IsoSeq method of PacBio enables sequencing of full-length transcripts up to $15 \mathrm{~Kb}$ using SMRT sequencing, in turn eliminating computational transcript reconstruction and the need for a reference genome.

By circumventing the bias prone elements in regular RNA sequencing, i.e., reverse transcription and PCR amplification of cDNA, Nanopore's direct RNA-seq enables the direct detection of full-length RNA. This real-time single-molecule method is based on two adapters; (1) a poly(T)adaptor for recognition and binding of the polyadenylated messenger RNA, and (2) a pair of sequencing adaptors that ligate onto the overhang of the poly(T)adaptors and facilitate its capture by a nanopore (Garalde et al., 2018).

Rolling Circle Amplification to Concatemeric Consensus (R2C2) method enables to generate a consensus from a single sequence read with many copies of an original molecule: this approach has been used to accurately produce full-length RNA transcript isoforms (Volden et al., 2018).

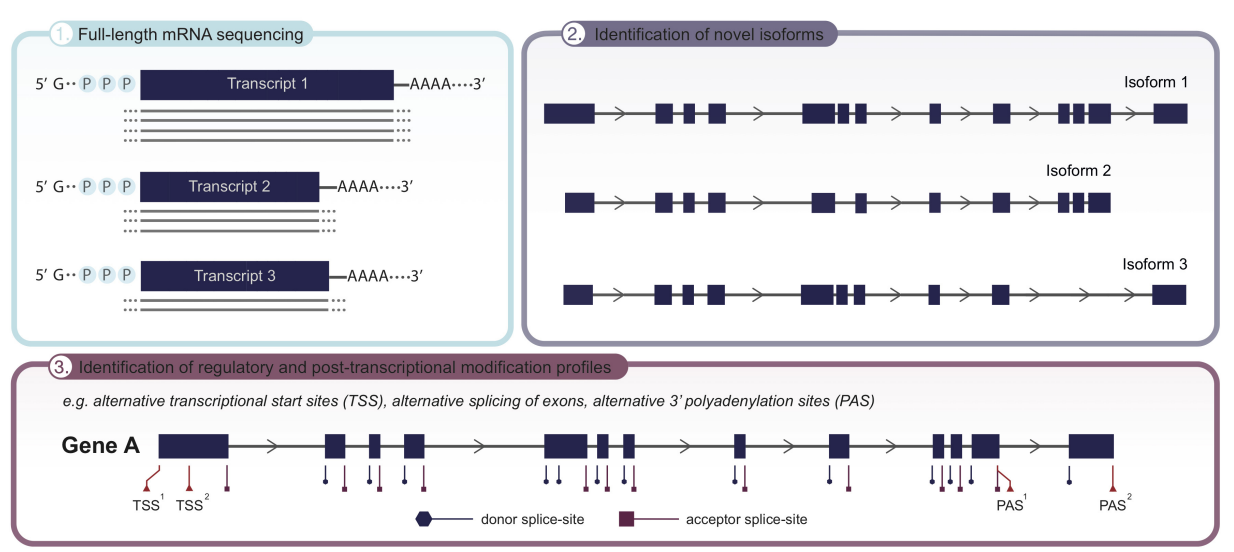

FIGURE 2 | Applicability of long-read sequencing (LRS) to unveil the transcriptome landscape of cells and tissues. Given the significant improvements in read length, employing LRS on RNA level now allows for full-length isoform sequencing, covering the complete mRNA transcript in one single read (panel 1). As recent advances have demonstrated the isoform landscape to be more complex than initially thought, LRS holds the potential to identify novel isoforms (panel 2), as well as detect transcriptional and post-transcriptional modification sites, e.g., alternative transcriptional start sites (TSS), alternative splicing of exons and alternative transcription termination sites ( $3^{\prime}$ polyadenylation sites; PAS), that underpin the emergence of different isoforms (panel 3). Collectively, uncovering the full isoform diversity within cells and tissues.

syndrome exhibiting revertant mosaicism, a rare phenomenon involving spontaneous correction of a pathogenic mutation by another mutation in a somatic cell. To keep in mind, Laver et al. (2016) pointed out problems in resolving allele phasing with targeted SMRT amplicon sequencing. In their study, the formation of chimeric molecules during PCR-amplification led to unambiguous results, which could have been avoided by using PCR-free methods (Ebbert et al., 2018; Hoijer et al., 2018). 
However, these methods can be costly and time consuming and simply keeping the amount of PCR cycles as low as possible could offer an easier solution (Laver et al., 2016).

Identification of SVs may also benefit from phasing (Seo et al., 2016; Cretu Stancu et al., 2017), in particular SV calling in cancer genomes can be significantly improved to understand the complexity of chromosomal rearrangements (Nattestad et al., 2018). Furthermore, phasing with LRS can be used to reveal the subclonal heterogeneity in malignancies (Lode et al., 2018). For genome-wide association studies (GWAS), multi-allelic haplotype markers can provide superior power compared to single-SNP markers in mapping disease loci (Akey et al., 2001; Khankhanian et al., 2015). However, the implementation of haplotype-based GWAS is highly dependent on capability to phase alleles (He et al., 2011). Therefore, LRS could be utilized to fine-map genetic associations within regions of interest that have previously been identified by GWAS. In addition to these multiple situations where accurate phasing is of great importance, LRS technology and the emerging ultra-long reads $(>100 \mathrm{~kb}$ ) from latest LRS developments may eventually produce fully phased diploid genomes (Pendleton et al., 2015; Seo et al., 2016; Jain et al., 2018; Chaisson et al., 2015b), which can even originate from single cells (Hills et al., 2018). This would allow for research that fully leverages phase information in order to understand how phenotypes are influenced by unique haplotype combinations (Tewhey et al., 2011).

\section{Pseudogenes}

Pseudogenes usually originate from gene duplication or retrotransposition events and are defined as sequences that resemble known functional genes but cannot produce functional proteins (D'Errico et al., 2004). Notably, the GENCODE project has estimated that the human genome contains more than 14,000 pseudogenes (Pei et al., 2012) and many clinically relevant genes (such as PMS2, CYP2D6, CHEK2, SMN1, and PKD1) are known to have pseudogenes with high sequence homology (Mandelker et al., 2016). This has significant consequences for re-sequencing studies as pseudogenes may severely impair reliable variant identification in their functional counterparts (Claes and De Leeneer, 2014) and lead to false diagnostic test results (Bardaro et al., 2003). Traditional methods, such as SR-NGS may capture sequences from pseudogenes in addition to the functional genes, leading to mapping errors, low variant detection rates and high number of false positives (Claes and De Leeneer, 2014). Other issues originate from the fact that some pseudogene sequences are still missing from the human reference genome and may cause mapping issues for SR-NGS (Karakoc et al., 2011). Therefore, assays based on SR-NGS may not always be reliable for investigating genes that have highly homologous pseudogenes.

To date, several studies (Table 1) have utilized LRS in order to investigate clinically relevant genes with homologous pseudogenes (Ammar et al., 2015; Qiao et al., 2016; Borras et al., 2017; Buermans et al., 2017; Frans et al., 2018). These studies have largely relied on target enrichment with long-range PCR (LR-PCR) using primers that locate to the rare mismatch sites that distinguish the gene of interest from its pseudogenes
(Frans et al., 2018). Similar approaches have been used with Sanger sequencing and SR-NGS. However, with LRS, the reads can span the complete LR-PCR amplicon and retain the phase information (Buermans et al., 2017). Furthermore, referencefree assembly of long reads can circumvent errors that may be introduced by complex or repetitive regions in the reference genome (Borras et al., 2017). For example, Polycystin 1 (PKD1), responsible for autosomal dominant polycystic kidney disease, has traditionally been challenging to analyze with NGS or Sanger sequencing because of its high GC-content, large size and homology with pseudogenes (Tan et al., 2014). Borras et al. (2017) demonstrated that by coupling LR-PCR with SMRT, the interference of residual PKD1 pseudogene amplification and alignment ambiguities could be eliminated, and SVs could be identified. In addition, multiple studies have demonstrated the utility of LRS for genotyping and phasing CYP2D6, which is one of the most important genes involved in drug metabolism, but has been difficult to analyze by conventional methods due to homologous pseudogenes and complex gene rearrangements (Ammar et al., 2015; Qiao et al., 2016; Seo et al., 2016; Buermans et al., 2017).

For medical genetics research, it is crucial to have reliable information on the population frequencies of particular gene variants in order to assess their potential pathogenicity (Ashley, 2016). For this, databases such as ExAC and GnomAD are often used (Lek et al., 2016). However, reference datasets attained with SR-NGS might be problematic for genes with highly homologous pseudogenes. This is mainly because: (1) the deposited variants may be derived from pseudogenes, (2) true variants might stay undetected or (3) the gene of interest might be ambiguously covered (Mandelker et al., 2016). Long reads could be used to improve the annotation of duplicated regions because they harbor sufficient number of paralogous sequence variants to confidently assign them to their respective paralogs (Dougherty et al., 2018). Therefore, in addition to more reliable pseudogene discrimination, LRS can be used to generate more accurate reference datasets and annotations for many challenging genes, subsequently allowing improvements in variant interpretation.

\section{UNCOVERING THE TRANSCRIPTOME LANDSCAPE TO AID GENETIC DIAGNOSIS}

The emergence of NGS, together with the incessancy of technological advances, drives our increasing ability to uncover complex and previously 'hidden' genetic aberrations, however, our current capacity to interpret their functional and clinical impact remains restrained. By providing a global transcriptomic profile, both quantitatively, i.e., gene expression levels, and qualitatively, i.e., transcript sequences and isoform structures, RNA sequencing holds the potential to reduce this diagnostic gap significantly. Recently two research groups pioneered this technology as a complementary tool to DNA-based tests and demonstrated its value in the detection of both coding and non-coding variants (Cummings et al., 2017; Kremer et al., 2017). In particular, authors identified a variety of variants 
underpinning (1) abnormal or mono-allelic expression and (2) aberrant splicing, resulting in the creation, skipping and truncation of exons, as well as exon and intron retention, even in regions harbouring repetitive regions. In turn, resulting in a diagnostic yield ranging from $10 \%$ for mitochondriopathies to $21 \%$ for primary muscle disorders in patients lacking strong candidates from WES or WGS (Cummings et al., 2017; Kremer et al., 2017).

Similar to NGS-based genomic approaches, however, shortread RNA sequencing methodologies are limited by their need for computational reconstruction of individual short reads into complete transcripts (Steijger et al., 2013). With the advent of long-read RNA sequencing the accurate identification and quantification of full-length mRNA transcript isoforms has become possible (Figure 2). A comparative approach by Anvar et al. (2018) has revealed up to $17 \%$ of novel alternative exons to be detected following long-read RNA sequencing of three primary human tissues, i.e., brain, heart, liver, thereby corroborating the isoform landscape to be more complex than initially thought (Sharon et al., 2013; Tilgner et al., 2013). In addition, the authors demonstrate the applicability of fulllength mRNA sequencing to uncover transcriptional regulation and post-translational modification profiles, including alternative transcription initiation, allele specific alternative splicing and alternative $3^{\prime}$ termination (polyadenylation) (Anvar et al., 2018). The clinical significance of integrating this transcriptomic data was exemplified by Aneichyk et al. (2018), who identified an intronic retrotransposon to induce alternative splicing that subsequently affects TAF1 expression, in turn causing X-linked Dystonia-Parkinsonism. Moreover, by exploring long-read RNA sequencing in the characterization of premature termination codons in $A B C A 7$ in late-onset Alzheimer's disease, De Roeck et al. (2017) observed various degrees of nonsense-mediated decay (NMD) and transcript modification that potentially influence $A B C A 7$ dosage and disease severity. While the ultimate goal may be to employ this technique to allocate the causative variant and map its functional impact on a patient-level, there may already be a major improvement by sequencing reference sets of tissue/cell specific RNA-isoform landscapes. Gaining insight into the isoform landscape of disease relevant tissues or cell types, potentially even on a single-cell level (Byrne et al., 2017; Gupta et al., 2018), will advance the overall interpretation of clinical significance of complex rearrangements (Nattestad et al., 2018), tissue-specific isoforms (Clark et al., 2018) and splice variants (de Jong et al., 2017). Ultimately, this knowledge can be implemented to improve WES/WGS-based variant filtering, prioritization and prediction of their functional impact.

\section{FUTURE PERSPECTIVES AND CONCLUSION}

Most of the clinically relevant examples described in this review use targeted LRS approaches, indicating that the broader use of LRS could significantly increase the diagnostic yield of genetic testing and discover novel disease genes. Especially SVs, repetitive elements and complex genomic regions that are difficult to assess with short reads can now be better assessed. LRS technology is also changing our view on the mRNA isoform landscape of different tissues and genes, potentially enabling better functional interpretation of genomic variation in the future. It is noted that, while the currently used LRS technologies are not quite yet at the stage of individual fully phased de novo assembled genomes, but with increased throughput, higher accuracy and lower costs, LRS-based WGS is coming within reach. Once this is routine, we are foreseeing the possibility that WGS with long reads could serve as a truly generic test that enables to detect all genetic variants present in an individual's genome. Then the power of LRS to overcome the current limitations in medical genetics may be elucidated at fast pace. Moreover, systematic studies utilizing LRS in patient cohorts with unsolved genetic diseases and control populations are warranted, and several consortia, e.g., www.solve-rd.eu or http://www.internationalgenome.org/ 1000-genomes-browsers, have already announced the use of LRS-WGS for this.

While the medical genetics community is now starting to realize the potential of this technology, some obstacles need to be overcome in order for LRS to be established as a mainstream tool (for overview of LRS limitations, see Box 1). Especially library preparation and analysis still need to reach the high level of robustness of SR-NGS technologies. For the data analysis, bioinformatic tools have traditionally been optimized for short-read sequencing data and need to be adapted, as well as thoroughly tested, for long reads (Sedlazeck et al., 2018a). In addition, analyzing whole genomes with LRS is still very expensive and the future application will depend largely on pricing progress of all NGS technologies, and may also depend on how fast the short-reads can reach an even lower prices with even better quality (i.e., the $\sim 100 \$$ genome). Eventually, one might need to consider a choice between sequencing many lessexpensive genomes with short-reads as opposed to investigating a smaller number of genomes in-detail using long reads. It is not a straightforward decision to make, however, the latter option would ideally be beneficial for an individual patient in clinical diagnostics. Combining SR-NGS and LRS can also be a powerful approach for highly accurate variant calling and assembly (Chaisson et al., 2019) and become increasingly important and more easily available with the planned acquisition of PacBio by Illumina.

We foresee that both of the prevailing LRS technologies, PacBio and ONT, will have a major influence on the future of medical genetics. ONT has the potential to become low in price, ease of use technology that is capable of directly investigating both native DNA and RNA in a high-throughput manner. The main challenge for ONT technology is to circumvent the systematic error rate, e.g., for homopolymer regions, which may be a limitation especially for future diagnostic applications. ONT has put forward ideas to improve this by optimizing bioinformatic tools that allow more accurate repeat lengths measurements and proposed to leverage different types of nanopores. One possible improvement was already enabled by the rolling circle amplification to concatemeric consensus method (R2C2) (Volden et al., 2018). For SMRT sequencing, the consensus accuracy achieved is better, especially once 
intra-molecule consensus - in addition to the existing inter-molecule consensus - calling is also feasible for longinsert libraries. To enable human genome sequencing, throughput needs to go up. To this end, PacBio has recently announced to release a SMRT cell with 8 million ZMWs in 2019 ( $\sim 8$-fold increase) and other improvements may come from even longer read lengths. This would enable much higher throughput and reduction in sequencing costs. Altogether, it remains to be seen whether the quality of the LRS technologies is sufficient for genome-wide detection of small indels and SNVs. If so, PacBio and ONT will have a chance to become the platform for true WGS for population scale studies in the near future. Moreover, the native epigenetic modifications of the DNA are preserved in PCR-free single molecule sequencing, opening up possibilities for novel LRS-based applications to assess base modifications (Pham et al., 2016; Euskirchen et al., 2017).

In addition to already commercially available technologies discussed in this review, others are in active development and will hopefully be available for users in the future. These include novel nanopore-based technologies from Genia (now acquired by Roche) and Stratos Genomics (Carson and Wanunu, 2015; Fuller et al., 2016), or the Electronic NanoDevice sequencing (ENDSeq) from Roswell Biotechnologies (Pugliese et al., 2015). Other exciting technologies that may also provide long-read insights are, e.g., library-and amplification-free technology from Nanostring (Hyb \& Seq), nanochannel genome mapping technology from Bionano Genomics (Lam et al., 2012; Seo et al., 2016) and Strand-Seq that preserves long-range context of homologous chromosomes (Sanders et al., 2017; Ghareghani et al., 2018). Whether these

\section{REFERENCES}

Akey, J., Jin, L., and Xiong, M. (2001). Haplotypes vs single marker linkage disequilibrium tests: what do we gain? Eur. J. Hum. Genet. 9, 291-300. doi: 10.1038/sj.ejhg.5200619

Alkan, C., Coe, B. P., and Eichler, E. E. (2011). Genome structural variation discovery and genotyping. Nat. Rev. Genet. 12, 363-376. doi: 10.1038/nrg2958

Ameur, A., Che, H., Martin, M., Bunikis, I., Dahlberg, J., Hoijer, I., et al. (2018a). De novo assembly of two Swedish genomes reveals missing segments from the human GRCh38 reference and improves variant calling of population-scale sequencing data. Genes 9:E486. doi: 10.3390/genes9100486

Ameur, A., Kloosterman, W. P., and Hestand, M. S. (2018b). Single-molecule sequencing: towards clinical applications. Trends Biotechnol. 37, 72-85. doi: 10.1016/j.tibtech.2018.07.013

Ammar, R., Paton, T. A., Torti, D., Shlien, A., and Bader, G. D. (2015). Long read nanopore sequencing for detection of HLA and CYP2D6 variants and haplotypes. F1000Res. 4:17. doi: 10.12688/f1000research.6037.2

Aneichyk, T., Hendriks, W. T., Yadav, R., Shin, D., Gao, D., Vaine, C. A., et al. (2018). Dissecting the causal mechanism of X-linked dystonia-parkinsonism by integrating genome and transcriptome assembly. Cell 172, 897-909.e21. doi: 10.1016/j.cell.2018.02.011

Anvar, S. Y., Allard, G., Tseng, E., Sheynkman, G. M., de Klerk, E., Vermaat, M., et al. (2018). Full-length mRNA sequencing uncovers a widespread coupling between transcription initiation and mRNA processing. Genome Biol. 19:46. doi: 10.1186/s13059-018-1418-0

Ardui, S., Ameur, A., Vermeesch, J. R., and Hestand, M. S. (2018a). Single molecule real-time (SMRT) sequencing comes of age: applications and utilities for medical diagnostics. Nucleic Acids Res. 46, 2159-2168. doi: 10.1093/nar/gky066 or other technologies are complementary to the existing LRS approaches, or may be able to compete with or even replace PacBio and ONT remains to be seen.

To conclude, we are on the advent of next revolution in sequencing technology. Once the major obstacles regarding accuracy, analysis and pricing are overcome, the time would be right to move towards individual fully phased long-read genomes, likely based on individual de novo assemblies that enable the identification of all the genetic variants regardless of their type.

\section{AUTHOR CONTRIBUTIONS}

$\mathrm{AH}$ and TM conceived the study. TM, SK, and AH drafted manuscript. SK prepared the figures.

\section{FUNDING}

AH was supported by the Solve-RD project. The Solve-RD project has received funding from the European Union's Horizon 2020 Research and Innovation Programme under grant agreement No. 779257. TM was supported by the Sigrid Jusélius Foundation. The Radboud Institute for Molecular Life Sciences supported AH and SK.

\section{ACKNOWLEDGMENTS}

The authors thank the funding agencies for their support and all colleagues for useful discussions.

Ardui, S., Race, V., de Ravel, T., Van Esch, H., Devriendt, K., Matthijs, G., et al. (2018b). Detecting AGG interruptions in females with a FMR1 premutation by long-read single-molecule sequencing: a 1 year clinical experience. Front. Genet. 9:150. doi: 10.3389/fgene.2018.00150

Ardui, S., Race, V., Zablotskaya, A., Hestand, M. S., Van Esch, H., Devriendt, K., et al. (2017). Detecting AGG interruptions in male and female FMR1 premutation carriers by single-molecule sequencing. Hum. Mutat. 38, 324-331. doi: 10.1002/humu. 23150

Ashley, E. A. (2016). Towards precision medicine. Nat. Rev. Genet. 17, 507-522. doi: 10.1038/nrg.2016.86

Bardaro, T., Falco, G., Sparago, A., Mercadante, V., Gean Molins, E., Tarantino, E., et al. (2003). Two cases of misinterpretation of molecular results in incontinentia pigmenti, and a PCR-based method to discriminate NEMO/IKKgamma dene deletion. Hum. Mutat. 21, 8-11. doi: 10.1002/humu. 10150

Benjamini, Y., and Speed, T. P. (2012). Summarizing and correcting the GC content bias in high-throughput sequencing. Nucleic Acids Res. 40:e72. doi: 10.1093/nar/ gks001

Borras, D. M., Vossen, R., Liem, M., Buermans, H. P. J., Dauwerse, H., van Heusden, D., et al. (2017). Detecting PKD1 variants in polycystic kidney disease patients by single-molecule long-read sequencing. Hum. Mutat. 38, 870-879. doi: 10.1002/humu.23223

Buermans, H. P., Vossen, R. H., Anvar, S. Y., Allard, W. G., Guchelaar, H. J., White, S. J., et al. (2017). Flexible and scalable full-length CYP2D6 long amplicon PacBio sequencing. Hum. Mutat. 38, 310-316. doi: 10.1002/humu. 23166

Butler, T. Z., Pavlenok, M., Derrington, I. M., Niederweis, M., and Gundlach, J. H. (2008). Single-molecule DNA detection with an engineered MspA protein 
nanopore. Proc. Natl. Acad. Sci. U.S.A. 105, 20647-20652. doi: 10.1073/pnas. 0807514106

Byrne, A., Beaudin, A. E., Olsen, H. E., Jain, M., Cole, C., Palmer, T., et al. (2017). Nanopore long-read RNAseq reveals widespread transcriptional variation among the surface receptors of individual B cells. Nat. Commun. 8:16027. doi: $10.1038 /$ ncomms 16027

Carneiro, M. O., Russ, C., Ross, M. G., Gabriel, S. B., Nusbaum, C., and DePristo, M. A. (2012). Pacific biosciences sequencing technology for genotyping and variation discovery in human data. BMC Genomics 13:375. doi: 10.1186/14712164-13-375

Carson, S., and Wanunu, M. (2015). Challenges in DNA motion control and sequence readout using nanopore devices. Nanotechnology 26:074004. doi: 10 . 1088/0957-4484/26/7/074004

Chaisson, M. J., Huddleston, J., Dennis, M. Y., Sudmant, P. H., Malig, M., Hormozdiari, F., et al. (2015a). Resolving the complexity of the human genome using single-molecule sequencing. Nature 517, 608-611. doi: 10.1038/ nature 13907

Chaisson, M. J., Wilson, R. K., and Eichler, E. E. (2015b). Genetic variation and the de novo assembly of human genomes. Nat. Rev. Genet. 16, 627-640. doi: $10.1038 / \mathrm{nrg} 3933$

Chaisson, M. J. P., Sanders, A. D., Zhao, X., Malhotra, A., Porubsky, D., Rausch, T., et al. (2019). Multi-platform discovery of haplotype-resolved structural variation in human genomes. Nat. Commun. 10:1784. doi: 10.1038/ s41467-018-08148-z

Chen, W., Ullmann, R., Langnick, C., Menzel, C., Wotschofsky, Z., Hu, H., et al. (2010). Breakpoint analysis of balanced chromosome rearrangements by next-generation paired-end sequencing. Eur. J. Hum. Genet. 18, 539-543. doi: 10.1038/ejhg.2009.211

Claes, K. B., and De Leeneer, K. (2014). Dealing with pseudogenes in molecular diagnostics in the next-generation sequencing era. Methods Mol. Biol. 1167, 303-315. doi: 10.1007/978-1-4939-0835-6_21

Clark, M., Wrzesinski, T., Garcia-Bea, A., Kleinman, J., Hyde, T., Weinberger, D., et al. (2018). Long-read sequencing reveals the splicing profile of the calcium channel gene CACNA1C in human brain. bioRxiv [Preprint]. doi: 10.1101/ 260562

Clarke, J., Wu, H. C., Jayasinghe, L., Patel, A., Reid, S., and Bayley, H. (2009). Continuous base identification for single-molecule nanopore DNA sequencing. Nat. Nanotechnol. 4, 265-270. doi: 10.1038/nnano.2009.12

Cretu Stancu, M., van Roosmalen, M. J., Renkens, I., Nieboer, M. M., Middelkamp, S., de Ligt, J., et al. (2017). Mapping and phasing of structural variation in patient genomes using nanopore sequencing. Nat. Commun. 8:1326. doi: 10 . 1038/s41467-017-01343-4

Cumming, S. A., Hamilton, M. J., Robb, Y., Gregory, H., McWilliam, C., Cooper, A., et al. (2018). De novo repeat interruptions are associated with reduced somatic instability and mild or absent clinical features in myotonic dystrophy type 1 . Eur. J. Hum. Genet. 26, 1635-1647. doi: 10.1038/s41431-018-0156-9

Cummings, B. B., Marshall, J. L., Tukiainen, T., Lek, M., Donkervoort, S., Foley, A. R., et al. (2017). Improving genetic diagnosis in Mendelian disease with transcriptome sequencing. Sci. Transl. Med. 9:eaal5209. doi: 10.1126/ scitranslmed.aal5209

Dastidar, S., Ardui, S., Singh, K., Majumdar, D., Nair, N., Fu, Y., et al. (2018). Efficient CRISPR/Cas9-mediated editing of trinucleotide repeat expansion in myotonic dystrophy patient-derived iPS and myogenic cells. Nucleic Acids Res. 46, 8275-8298. doi: 10.1093/nar/gky548

De Coster, W., De Roeck, A., De Pooter, T., D’Hert, S., De Rijk, P., Strazisar, M., et al. (2018). Structural variants identified by Oxford Nanopore PromethION sequencing of the human genome. bioRxiv [Preprint]. doi: 10.1101/434118

de Jong, L. C., Cree, S., Lattimore, V., Wiggins, G. A. R., Spurdle, A. B., kConFab Investigators, et al. (2017). Nanopore sequencing of full-length BRCA1 mRNA transcripts reveals co-occurrence of known exon skipping events. Breast Cancer Res. 19:127. doi: 10.1186/s13058-017-0919-1

de Koning, A. P., Gu, W., Castoe, T. A., Batzer, M. A., and Pollock, D. D. (2011). Repetitive elements may comprise over two-thirds of the human genome. PLoS Genet. 7:e1002384. doi: 10.1371/journal.pgen.1002384

De Roeck, A., De Coster, W., Bossaerts, L., Cacace, R., De Pooter, T., Van Dongen, J., et al. (2018). Accurate characterization of expanded tandem repeat length and sequence through whole genome long-read sequencing on PromethION. bioRxiv
De Roeck, A., Van den Bossche, T., van der Zee, J., Verheijen, J., De Coster, W., Van Dongen, J., et al. (2017). Deleterious ABCA7 mutations and transcript rescue mechanisms in early onset Alzheimer's disease. Acta Neuropathol. 134, 475-487. doi: 10.1007/s00401-017-1714-x

Deamer, D., Akeson, M., and Branton, D. (2016). Three decades of nanopore sequencing. Nat. Biotechnol. 34, 518-524. doi: 10.1038/nbt.3423

Delaneau, O., Howie, B., Cox, A. J., Zagury, J. F., and Marchini, J. (2013). Haplotype estimation using sequencing reads. Am. J. Hum. Genet. 93, 687-696. doi: 10. 1016/j.ajhg.2013.09.002

D’Errico, I., Gadaleta, G., and Saccone, C. (2004). Pseudogenes in metazoa: origin and features. Brief. Funct. Genomic Proteomic 3, 157-167. doi: 10.1093/bfgp/3. 2.157

Dimitriadou, E., Melotte, C., Debrock, S., Esteki, M. Z., Dierickx, K., Voet, T., et al. (2017). Principles guiding embryo selection following genome-wide haplotyping of preimplantation embryos. Hum. Reprod. 32, 687-697. doi: 10. 1093/humrep/dex011

Dolzhenko, E., van Vugt, J., Shaw, R. J., Bekritsky, M. A., van Blitterswijk, M., Narzisi, G., et al. (2017). Detection of long repeat expansions from PCR-free whole-genome sequence data. Genome Res. 27, 1895-1903. doi: 10.1101/gr. 225672.117

Dougherty, M. L., Underwood, J. G., Nelson, B. J., Tseng, E., Munson, K. M., Penn, O., et al. (2018). Transcriptional fates of human-specific segmental duplications in brain. Genome Res. 28, 1566-1576. doi: 10.1101/gr.237610.118

Dutta, U. R., Rao, S. N., Pidugu, V. K., Vineeth, V. S., Bhattacherjee, A., Bhowmik, A. D., et al. (2018). Breakpoint mapping of a novel de novo translocation $\mathrm{t}(\mathrm{X} ; 20)(\mathrm{q} 11.1 ; \mathrm{p} 13)$ by positional cloning and long read sequencing. Genomics [Epub ahead of print].

Ebbert, M. T. W., Farrugia, S. L., Sens, J. P., Jansen-West, K., Gendron, T. F., Prudencio, M., et al. (2018). Long-read sequencing across the C9orf72 'GGGGCC' repeat expansion: implications for clinical use and genetic discovery efforts in human disease. Mol. Neurodegener. 13:46. doi: 10.1186/s13024-0180274-4

Eid, J., Fehr, A., Gray, J., Luong, K., Lyle, J., Otto, G., et al. (2009). Real-time DNA sequencing from single polymerase molecules. Science 323, 133-138. doi: 10.1126/science.1162986

Euskirchen, P., Bielle, F., Labreche, K., Kloosterman, W. P., Rosenberg, S., Daniau, M., et al. (2017). Same-day genomic and epigenomic diagnosis of brain tumors using real-time nanopore sequencing. Acta Neuropathol. 134, 691-703. doi: 10.1007/s00401-017-1743-5

Fan, H. C., Wang, J., Potanina, A., and Quake, S. R. (2011). Whole-genome molecular haplotyping of single cells. Nat. Biotechnol. 29, 51-57. doi: 10.1038/ nbt.1739

Feuk, L., Carson, A. R., and Scherer, S. W. (2006). Structural variation in the human genome. Nat. Rev. Genet. 7, 85-97. doi: 10.1038/nrg1767

Flusberg, B. A., Webster, D. R., Lee, J. H., Travers, K. J., Olivares, E. C., Clark, T. A., et al. (2010). Direct detection of DNA methylation during single-molecule, real-time sequencing. Nat. Methods 7, 461-465. doi: 10.1038/nmeth.1459

Frans, G., Meert, W., Van der Werff Ten Bosch, J., Meyts, I., Bossuyt, X., Vermeesch, J. R., et al. (2018). Conventional and single-molecule targeted sequencing method for specific variant detection in IKBKG while bypassing the IKBKGP1 pseudogene. J. Mol. Diagn. 20, 195-202. doi: 10.1016/j.jmoldx.2017. 10.005

Fuller, C. W., Kumar, S., Porel, M., Chien, M., Bibillo, A., Stranges, P. B., et al. (2016). Real-time single-molecule electronic DNA sequencing by synthesis using polymer-tagged nucleotides on a nanopore array. Proc. Natl. Acad. Sci. U.S.A. 113, 5233-5238. doi: 10.1073/pnas.1601782113

Gabrieli, T., Sharim, H., Fridman, D., Arbib, N., Michaeli, Y., and Ebenstein, Y. (2018). Selective nanopore sequencing of human BRCA1 by Cas9-assisted targeting of chromosome segments (CATCH). Nucleic Acids Res. 46:e87. doi: 10.1093/nar/gky411

Garalde, D. R., Snell, E. A., Jachimowicz, D., Sipos, B., Lloyd, J. H., Bruce, M., et al. (2018). Highly parallel direct RNA sequencing on an array of nanopores. Nat. Methods 15, 201-206. doi: 10.1038/nmeth.4577

Genomes Project Consortium, Abecasis, G. R., Auton, A., Brooks, L. D., DePristo, M. A., Durbin, R. M., et al. (2012). An integrated map of genetic variation from 1,092 human genomes. Nature 491, 56-65. doi: 10.1038/nature11632

Genovese, G., Handsaker, R. E., Li, H., Altemose, N., Lindgren, A. M., Chambert, K., et al. (2013). Using population admixture to help complete 
maps of the human genome. Nat. Genet. 45, e401-e402. doi: 10.1038/ng. 2565

Ghareghani, M., Porubsky, D., Sanders, A. D., Meiers, S., Eichler, E. E., Korbel, J. O., et al. (2018). Strand-seq enables reliable separation of long reads by chromosome via expectation maximization. Bioinformatics 34, i115-i123. doi: 10.1093/bioinformatics/bty290

Gilissen, C., Hehir-Kwa, J. Y., Thung, D. T., van de Vorst, M., van Bon, B. W., Willemsen, M. H., et al. (2014). Genome sequencing identifies major causes of severe intellectual disability. Nature 511, 344-347. doi: 10.1038/nature 13394

Gilissen, C., Hoischen, A., Brunner, H. G., and Veltman, J. A. (2011). Unlocking Mendelian disease using exome sequencing. Genome Biol. 12:228. doi: 10.1186/ gb-2011-12-9-228

Goldmann, J. M., Wong, W. S., Pinelli, M., Farrah, T., Bodian, D., Stittrich, A. B., et al. (2016). Parent-of-origin-specific signatures of de novo mutations. Nat. Genet. 48, 935-939. doi: 10.1038/ng.3597

Goodwin, S., McPherson, J. D., and McCombie, W. R. (2016). Coming of age: ten years of next-generation sequencing technologies. Nat. Rev. Genet. 17, 333-351. doi: 10.1038/nrg.2016.49

Gudmundsson, S., Wilbe, M., Ekvall, S., Ameur, A., Cahill, N., Alexandrov, L. B., et al. (2017). Revertant mosaicism repairs skin lesions in a patient with keratitisichthyosis-deafness syndrome by second-site mutations in connexin 26. Hum. Mol. Genet. 26, 1070-1077. doi: 10.1093/hmg/ddx017

Gupta, I., Collier, P. G., Haase, B., Mahfouz, A., Joglekar, A., Floyd, T., et al. (2018). Single-cell isoform RNA sequencing characterizes isoforms in thousands of cerebellar cells. Nat. Biotechnol. 36, 1197-1202. doi: 10.1038/nbt.4259

He, Y., Li, C., Amos, C. I., Xiong, M., Ling, H., and Jin, L. (2011). Accelerating haplotype-based genome-wide association study using perfect phylogeny and phase-known reference data. PLoS One 6:e22097. doi: 10.1371/journal.pone. 0022097

Hebert, P. D. N., Braukmann, T. W. A., Prosser, S. W. J., Ratnasingham, S., deWaard, J. R., Ivanova, N. V., et al. (2018). A Sequel to Sanger: amplicon sequencing that scales. BMC Genomics 19:219. doi: 10.1186/s12864-0184611-3

Hestand, M. S., Van Houdt, J., Cristofoli, F., and Vermeesch, J. R. (2016). Polymerase specific error rates and profiles identified by single molecule sequencing. Mutat. Res. 784-785, 39-45. doi: 10.1016/j.mrfmmm.2016. 01.003

Hills, M., Falconer, E., O’Neil, K., Sanders, A., Howe, K., Guryev, V., et al. (2018). Construction of whole genomes from scaffolds using single cell STRAND-SEQ data. bioRxiv [Preprint]. doi: 10.1101/271510

Hoijer, I., Tsai, Y. C., Clark, T. A., Kotturi, P., Dahl, N., Stattin, E. L., et al. (2018). Detailed analysis of HTT repeat elements in human blood using targeted amplification-free long-read sequencing. Hum. Mutat. 39, 1262-1272. doi: 10. 1002/humu. 23580

Huddleston, J., Chaisson, M. J. P., Steinberg, K. M., Warren, W., Hoekzema, K., Gordon, D., et al. (2017). Discovery and genotyping of structural variation from long-read haploid genome sequence data. Genome Res. 27, 677-685. doi: 10.1101/gr.214007.116

Ishiura, H., Doi, K., Mitsui, J., Yoshimura, J., Matsukawa, M. K., Fujiyama, A., et al. (2018). Expansions of intronic TTTCA and TTTTA repeats in benign adult familial myoclonic epilepsy. Nat. Genet. 50, 581-590. doi: 10.1038/s41588-0180067-2

Jain, M., Fiddes, I. T., Miga, K. H., Olsen, H. E., Paten, B., and Akeson, M. (2015). Improved data analysis for the MinION nanopore sequencer. Nat. Methods 12 , 351-356. doi: 10.1038/nmeth.3290

Jain, M., Koren, S., Miga, K. H., Quick, J., Rand, A. C., Sasani, T. A., et al. (2018). Nanopore sequencing and assembly of a human genome with ultra-long reads. Nat. Biotechnol. 36, 338-345. doi: 10.1038/nbt.4060

Jiang, W., Zhao, X., Gabrieli, T., Lou, C., Ebenstein, Y., and Zhu, T. F. (2015). Cas9-Assisted Targeting of CHromosome segments CATCH enables one-step targeted cloning of large gene clusters. Nat. Commun. 6:8101. doi: 10.1038/ ncomms 9101

Karakoc, E., Alkan, C., O’Roak, B. J., Dennis, M. Y., Vives, L., Mark, K., et al. (2011). Detection of structural variants and indels within exome data. Nat. Methods 9, 176-178. doi: 10.1038/nmeth. 1810

Kasianowicz, J. J., Brandin, E., Branton, D., and Deamer, D. W. (1996). Characterization of individual polynucleotide molecules using a membrane channel. Proc. Natl. Acad. Sci. U.S.A. 93, 13770-13773. doi: 10.1073/pnas.93. 24.13770

Khankhanian, P., Gourraud, P. A., Lizee, A., and Goodin, D. S. (2015). Haplotypebased approach to known MS-associated regions increases the amount of explained risk. J. Med. Genet. 52, 587-594. doi: 10.1136/jmedgenet-2015103071

Kitzman, J. O., Mackenzie, A. P., Adey, A., Hiatt, J. B., Patwardhan, R. P., Sudmant, P. H., et al. (2011). Haplotype-resolved genome sequencing of a Gujarati Indian individual. Nat. Biotechnol. 29, 59-63. doi: 10.1038/nbt.1740

Kremer, E. J., Pritchard, M., Lynch, M., Yu, S., Holman, K., Baker, E., et al. (1991). Mapping of DNA instability at the fragile $\mathrm{X}$ to a trinucleotide repeat sequence p(CCG)n. Science 252, 1711-1714. doi: 10.1126/science.1675488

Kremer, L. S., Bader, D. M., Mertes, C., Kopajtich, R., Pichler, G., Iuso, A., et al. (2017). Genetic diagnosis of Mendelian disorders via RNA sequencing. Nat. Commun. 8:15824. doi: 10.1038/ncomms15824

Krishnakumar, R., Sinha, A., Bird, S. W., Jayamohan, H., Edwards, H. S., Schoeniger, J. S., et al. (2018). Systematic and stochastic influences on the performance of the MinION nanopore sequencer across a range of nucleotide bias. Sci. Rep. 8:3159. doi: 10.1038/s41598-018-21484-w

Kuleshov, V., Xie, D., Chen, R., Pushkarev, D., Ma, Z., Blauwkamp, T., et al. (2014). Whole-genome haplotyping using long reads and statistical methods. Nat. Biotechnol. 32, 261-266. doi: 10.1038/nbt.2833

Lam, E. T., Hastie, A., Lin, C., Ehrlich, D., Das, S. K., Austin, M. D., et al. (2012). Genome mapping on nanochannel arrays for structural variation analysis and sequence assembly. Nat. Biotechnol. 30, 771-776. doi: 10.1038/nbt.2303

Laszlo, A. H., Derrington, I. M., Brinkerhoff, H., Langford, K. W., Nova, I. C., Samson, J. M., et al. (2013). Detection and mapping of 5-methylcytosine and 5-hydroxymethylcytosine with nanopore MspA. Proc. Natl. Acad. Sci. U.S.A. 110, 18904-18909. doi: 10.1073/pnas.1310240110

Laver, T. W., Caswell, R. C., Moore, K. A., Poschmann, J., Johnson, M. B., Owens, M. M., et al. (2016). Pitfalls of haplotype phasing from amplicon-based long-read sequencing. Sci. Rep. 6:21746. doi: 10.1038/srep21746

Lee, H., Gurtowski, J., Yoo, S., Nattestad, M., Marcus, S., Goodwin, S., et al. (2016). Third-generation sequencing and the future of genomics. bioRxiv [Preprint]. doi: 10.1101/048603

Lek, M., Karczewski, K. J., Minikel, E. V., Samocha, K. E., Banks, E., Fennell, T., et al. (2016). Analysis of protein-coding genetic variation in 60,706 humans. Nature 536, 285-291. doi: 10.1038/nature19057

Lemmers, R. J., van der Vliet, P. J., Balog, J., Goeman, J. J., Arindrarto, W., Krom, Y. D., et al. (2018). Deep characterization of a common D4Z4 variant identifies biallelic DUX4 expression as a modifier for disease penetrance in FSHD2. Eur. J. Hum. Genet. 26, 94-106. doi: 10.1038/s41431-017-0015-0

Levene, M. J., Korlach, J., Turner, S. W., Foquet, M., Craighead, H. G., and Webb, W. W. (2003). Zero-mode waveguides for single-molecule analysis at high concentrations. Science 299, 682-686. doi: 10.1126/science. 1079700

Lode, L., Ameur, A., Coste, T., Menard, A., Richebourg, S., Gaillard, J. B., et al. (2018). Single-molecule DNA sequencing of acute myeloid leukemia and myelodysplastic syndromes with multiple TP53 alterations. Haematologica 103, e13-e16. doi: 10.3324/haematol.2017.176719

Loomis, E. W., Eid, J. S., Peluso, P., Yin, J., Hickey, L., Rank, D., et al. (2013). Sequencing the unsequenceable: expanded CGG-repeat alleles of the fragile $\mathrm{X}$ gene. Genome Res. 23, 121-128. doi: 10.1101/gr.141705.112

Loose, M., Malla, S., and Stout, M. (2016). Real-time selective sequencing using nanopore technology. Nat. Methods 13, 751-754. doi: 10.1038/nmeth.3930

Mandelker, D., Schmidt, R. J., Ankala, A., McDonald Gibson, K., Bowser, M., Sharma, H., et al. (2016). Navigating highly homologous genes in a molecular diagnostic setting: a resource for clinical next-generation sequencing. Genet. Med. 18, 1282-1289. doi: 10.1038/gim.2016.58

Marks, P., Garcia, S., Barrio, A. M., Belhocine, K., Bernate, J., Bharadwaj, R., et al. (2019). Resolving the full spectrum of human genome variation using Linked-Reads. Genome Res. 29, 635-645. doi: 10.1101/gr.234443.118

Marks, P., Garcia, S., Barrio, A. M., Belhocine, K., Bernate, J., Bharadwaj, R., et al. (2019). Resolving the full spectrum of human genome variation using linked-reads. Genome Res. 29, 635-645. doi: 10.1101/gr.234443.118

McFarland, K. N., Liu, J., Landrian, I., Godiska, R., Shanker, S., Yu, F., et al. (2015). SMRT sequencing of long tandem nucleotide repeats in SCA10 reveals unique insight of repeat expansion structure. PLoS One 10:e0135906. doi: 10.1371/ journal.pone.0135906 
Menegon, M., Cantaloni, C., Rodriguez-Prieto, A., Centomo, C., Abdelfattah, A., Rossato, M., et al. (2017). On site DNA barcoding by nanopore sequencing. PLoS One 12:e184741. doi: 10.1371/journal.pone.0184741

Merker, J. D., Wenger, A. M., Sneddon, T., Grove, M., Zappala, Z., Fresard, L., et al. (2018). Long-read genome sequencing identifies causal structural variation in a Mendelian disease. Genet. Med. 20, 159-163. doi: 10.1038/gim.2017.86

Miao, H., Zhou, J., Yang, Q., Liang, F., Wang, D., Ma, N., et al. (2018). Longread sequencing identified a causal structural variant in an exome-negative case and enabled preimplantation genetic diagnosis. Hereditas 155:32. doi: 10.1186/ s41065-018-0069-1

Nattestad, M., Goodwin, S., Ng, K., Baslan, T., Sedlazeck, F. J., Rescheneder, P., et al. (2018). Complex rearrangements and oncogene amplifications revealed by long-read DNA and RNA sequencing of a breast cancer cell line. Genome Res. 28, 1126-1135. doi: 10.1101/gr.231100.117

Nazaryan-Petersen, L., Eisfeldt, J., Pettersson, M., Lundin, J., Nilsson, D., Wincent, J., et al. (2018). Replicative and non-replicative mechanisms in the formation of clustered CNVs are indicated by whole genome characterization. PLoS Genet. 14:e1007780. doi: 10.1371/journal.pgen.1007780

Neveling, K., Collin, R. W., Gilissen, C., van Huet, R. A., Visser, L., Kwint, M. P., et al. (2012). Next-generation genetic testing for retinitis pigmentosa. Hum. Mutat. 33, 963-972. doi: 10.1002/humu.22045

Nolin, S. L., Dobkin, C., and Brown, W. T. (2003). Molecular analysis of fragile X syndrome. Curr. Protoc. Hum. Genet. 63, 9.5.1-9.5.16. doi: 10.1002/ 0471142905.hg0905s38

Nolin, S. L., Glicksman, A., Ersalesi, N., Dobkin, C., Brown, W. T., Cao, R., et al. (2015). Fragile X full mutation expansions are inhibited by one or more AGG interruptions in premutation carriers. Genet. Med. 17, 358-364. doi: 10.1038/ gim.2014.106

Pei, B., Sisu, C., Frankish, A., Howald, C., Habegger, L., Mu, X. J., et al. (2012). The GENCODE pseudogene resource. Genome Biol. 13:R51. doi: 10.1186/gb-201213-9-r51

Pendleton, M., Sebra, R., Pang, A. W., Ummat, A., Franzen, O., Rausch, T., et al. (2015). Assembly and diploid architecture of an individual human genome via single-molecule technologies. Nat. Methods 12, 780-786. doi: 10.1038/nmeth. 3454

Peprah, E. (2012). Fragile X syndrome: the FMR1 CGG repeat distribution among world populations. Ann. Hum. Genet. 76, 178-191. doi: 10.1111/j.1469-1809. 2011.00694.x

Peters, B. A., Kermani, B. G., Sparks, A. B., Alferov, O., Hong, P., Alexeev, A., et al. (2012). Accurate whole-genome sequencing and haplotyping from 10 to 20 human cells. Nature 487, 190-195. doi: 10.1038/nature11236

Pham, T. T., Yin, J., Eid, J. S., Adams, E., Lam, R., Turner, S. W., et al. (2016). Singlelocus enrichment without amplification for sequencing and direct detection of epigenetic modifications. Mol. Genet. Genomics 291, 1491-1504. doi: 10.1007/ s00438-016-1167-2

Pollard, M. O., Gurdasani, D., Mentzer, A. J., Porter, T., and Sandhu, M. S. (2018). Long reads: their purpose and place. Hum. Mol. Genet. 27, R234-R241. doi: 10.1093/hmg/ddy177

Pugliese, K. M., Gul, O. T., Choi, Y., Olsen, T. J., Sims, P. C., Collins, P. G., et al. (2015). Processive incorporation of deoxynucleoside triphosphate analogs by single-molecule DNA polymerase I (klenow fragment) nanocircuits. J. Am. Chem. Soc. 137, 9587-9594. doi: 10.1021/jacs.5b02074

Qiao, W., Yang, Y., Sebra, R., Mendiratta, G., Gaedigk, A., Desnick, R. J., et al. (2016). Long-read single molecule real-time full gene sequencing of cytochrome P450-2D6. Hum. Mutat. 37, 315-323. doi: 10.1002/humu.22936

Quail, M. A., Smith, M., Coupland, P., Otto, T. D., Harris, S. R., Connor, T. R., et al. (2012). A tale of three next generation sequencing platforms: comparison of Ion Torrent, Pacific Biosciences and Illumina MiSeq sequencers. BMC Genomics 13:341. doi: 10.1186/1471-2164-13-341

Reiner, J., Pisani, L., Qiao, W., Singh, R., Yang, Y., Shi, L., et al. (2018). Cytogenomic identification and long-read single molecule real-time (SMRT) sequencing of a Bardet-Biedl Syndrome 9 (BBS9) deletion. NPJ Genom. Med. 3:3. doi: 10.1038/ s41525-017-0042-3

Roberts, R. J., Carneiro, M. O., and Schatz, M. C. (2013). The advantages of SMRT sequencing. Genome Biol. 14:405. doi: 10.1186/gb-2013-14-6-405

Salzberg, S. L., and Yorke, J. A. (2005). Beware of mis-assembled genomes. Bioinformatics 21, 4320-4321. doi: 10.1093/bioinformatics/bti769

Sanders, A. D., Falconer, E., Hills, M., Spierings, D. C. J., and Lansdorp, P. M. (2017). Single-cell template strand sequencing by Strand-seq enables the characterization of individual homologs. Nat. Protoc. 12, 1151-1176. doi: 10.1038/nprot.2017.029

Schadt, E. E., Turner, S., and Kasarskis, A. (2010). A window into thirdgeneration sequencing. Hum. Mol. Genet. 19, R227-R240. doi: 10.1093/hmg/ ddq416

Schule, B., McFarland, K. N., Lee, K., Tsai, Y. C., Nguyen, K. D., Sun, C., et al. (2017). Parkinson's disease associated with pure ATXN10 repeat expansion. NPJ Parkinsons Dis. 3:27. doi: 10.1038/s41531-017-0029-x

Sedlazeck, F. J., Lee, H., Darby, C. A., and Schatz, M. C. (2018a). Piercing the dark matter: bioinformatics of long-range sequencing and mapping. Nat. Rev. Genet. 19, 329-346. doi: 10.1038/s41576-018-0003-4

Sedlazeck, F. J., Rescheneder, P., Smolka, M., Fang, H., Nattestad, M., von Haeseler, A., et al. (2018b). Accurate detection of complex structural variations using single-molecule sequencing. Nat. Methods 15, 461-468. doi: 10.1038/s41592018-0001-7

Seo, J. S., Rhie, A., Kim, J., Lee, S., Sohn, M. H., Kim, C. U., et al. (2016). De novo assembly and phasing of a Korean human genome. Nature 538, 243-247. doi: 10.1038/nature20098

Sharon, D., Tilgner, H., Grubert, F., and Snyder, M. (2013). A single-molecule long-read survey of the human transcriptome. Nat. Biotechnol. 31, 1009-1014. doi: $10.1038 / \mathrm{nbt} .2705$

Shendure, J., Balasubramanian, S., Church, G. M., Gilbert, W., Rogers, J., Schloss, J. A., et al. (2017). DNA sequencing at 40: past, present and future. Nature 550, 345-353. doi: 10.1038/nature24286

Shi, L., Guo, Y., Dong, C., Huddleston, J., Yang, H., Han, X., et al. (2016). Long-read sequencing and de novo assembly of a Chinese genome. Nat. Commun. 7:12065. doi: $10.1038 /$ ncomms 12065

Song, J. H. T., Lowe, C. B., and Kingsley, D. M. (2018). Characterization of a human-specific tandem repeat associated with bipolar disorder and schizophrenia. Am. J. Hum. Genet. 103, 421-430. doi: 10.1016/j.ajhg.2018.07.011

Steijger, T., Abril, J. F., Engstrom, P. G., Kokocinski, F., Consortium, R., Hubbard, T. J., et al. (2013). Assessment of transcript reconstruction methods for RNAseq. Nat. Methods 10, 1177-1184. doi: 10.1038/nmeth.2714

Sudmant, P. H., Rausch, T., Gardner, E. J., Handsaker, R. E., Abyzov, A., Huddleston, J., et al. (2015). An integrated map of structural variation in 2,504 human genomes. Nature 526, 75-81. doi: 10.1038/nature15394

Tan, A. Y., Michaeel, A., Liu, G., Elemento, O., Blumenfeld, J., Donahue, S., et al. (2014). Molecular diagnosis of autosomal dominant polycystic kidney disease using next-generation sequencing. J. Mol. Diagn. 16, 216-228. doi: 10.1016/j. jmoldx.2013.10.005

Tang, H., Kirkness, E. F., Lippert, C., Biggs, W. H., Fabani, M., Guzman, E., et al. (2017). Profiling of short-tandem-repeat disease alleles in 12,632 human whole genomes. Am. J. Hum. Genet. 101, 700-715. doi: 10.1016/j.ajhg.2017. 09.013

Tattini, L., D'Aurizio, R., and Magi, A. (2015). Detection of Genomic structural variants from next-generation sequencing data. Front. Bioeng. Biotechnol. 3:92. doi: 10.3389/fbioe.2015.00092

Tewhey, R., Bansal, V., Torkamani, A., Topol, E. J., and Schork, N. J. (2011). The importance of phase information for human genomics. Nat. Rev. Genet. 12, 215-223. doi: 10.1038/nrg2950

Tilgner, H., Raha, D., Habegger, L., Mohiuddin, M., Gerstein, M., and Snyder, M. (2013). Accurate identification and analysis of human mRNA isoforms using deep long read sequencing. G3 3, 387-397. doi: 10.1534/g3.112.004812

Travers, K. J., Chin, C. S., Rank, D. R., Eid, J. S., and Turner, S. W. (2010). A flexible and efficient template format for circular consensus sequencing and SNP detection. Nucleic Acids Res. 38:e159. doi: 10.1093/nar/gkq543

Treangen, T. J., and Salzberg, S. L. (2011). Repetitive DNA and next-generation sequencing: computational challenges and solutions. Nat. Rev. Genet. 13, 36-46. doi: $10.1038 / \mathrm{nrg} 3117$

Tsai, Y.-C., Greenberg, D., Powell, J., Hoijer, I., Ameur, A., Strahl, M., et al. (2017). Amplification-free, CRISPR-Cas9 targeted enrichment and SMRT sequencing of repeat-expansion disease causative genomic regions. bioRxiv [Preprint]. doi: $10.1101 / 203919$

van Dijk, E. L., Jaszczyszyn, Y., Naquin, D., and Thermes, C. (2018). The third revolution in sequencing technology. Trends Genet. 34, 666-681. doi: 10.1016/ j.tig.2018.05.008

Volden, R., Palmer, T., Byrne, A., Cole, C., Schmitz, R. J., Green, R. E., et al. (2018). Improving nanopore read accuracy with the R2C2 method enables the 
sequencing of highly multiplexed full-length single-cell cDNA. Proc. Natl. Acad. Sci. U.S.A. 115, 9726-9731. doi: 10.1073/pnas. 1806447115

Wang, M., Beck, C. R., English, A. C., Meng, Q., Buhay, C., Han, Y., et al. (2015) PacBio-LITS: a large-insert targeted sequencing method for characterization of human disease-associated chromosomal structural variations. BMC Genomics 16:214. doi: 10.1186/s12864-015-1370-2

Weisenfeld, N. I., Kumar, V., Shah, P., Church, D. M., and Jaffe, D. B. (2017). Direct determination of diploid genome sequences. Genome Res. 27, 757-767. doi: $10.1101 /$ gr.214874.116

Wenger, A. M., Peluso, P., Rowell, W. J., Chang, P.-C., Hall, R. J., Concepcion, G. T., et al. (2019). Highly-accurate long-read sequencing improves variant detection and assembly of a human genome. bioRxiv [Preprint]. doi: 10.1101/519025

Wilbe, M., Gudmundsson, S., Johansson, J., Ameur, A., Stattin, E. L., Anneren, G., et al. (2017). A novel approach using long-read sequencing and ddPCR to investigate gonadal mosaicism and estimate recurrence risk in two families with developmental disorders. Prenat. Diagn. 37, 1146-1154. doi: 10.1002/pd.5156

Zamani Esteki, M., Dimitriadou, E., Mateiu, L., Melotte, C., Van der Aa, N., Kumar, P., et al. (2015). Concurrent whole-genome haplotyping and copy-number profiling of single cells. Am. J. Hum. Genet. 96, 894-912. doi: 10.1016/j.ajhg. 2015.04.011
Zeng, S., Zhang, M. Y., Wang, X. J., Hu, Z. M., Li, J. C., Li, N., et al. (2018). Long-read sequencing identified intronic repeat expansions in SAMD12 from Chinese pedigrees affected with familial cortical myoclonic tremor with epilepsy. J. Med. Genet. 56, 265-270. doi: 10.1136/jmedgenet-2018-10 5484

Zheng, G. X., Lau, B. T., Schnall-Levin, M., Jarosz, M., Bell, J. M., Hindson, C. M., et al. (2016). Haplotyping germline and cancer genomes with high-throughput linked-read sequencing. Nat. Biotechnol. 34, 303-311. doi: 10.1038/nbt. 3432

Conflict of Interest Statement: The authors declare that the research was conducted in the absence of any commercial or financial relationships that could be construed as a potential conflict of interest.

Copyright $\odot 2019$ Mantere, Kersten and Hoischen. This is an open-access article distributed under the terms of the Creative Commons Attribution License (CC BY). The use, distribution or reproduction in other forums is permitted, provided the original author(s) and the copyright owner(s) are credited and that the original publication in this journal is cited, in accordance with accepted academic practice. No use, distribution or reproduction is permitted which does not comply with these terms. 